\title{
The Clinicopathological Correlations of hTERC Amplification with Esophageal Squamous Cell Precursor Lesions
}

\author{
Yanping $\mathrm{Hu}^{1}{ }^{1} \cdot$ Xiaojing Teng ${ }^{2} \cdot$ Linlin $\mathrm{Wu}^{1} \cdot$ Wei Liu ${ }^{2}$ Jianduo $\mathrm{An}^{1}$
}

Received: 25 April 2018 / Accepted: 3 October 2018 / Published online: 11 October 2018

(c) The Author(s) 2018

\begin{abstract}
Background Esophageal squamous cell precursor lesions remain one of the most controversial topics in pathology and clinical management.

Aims To analyze the dysregulation of human telomerase RNA component (hTERC) in esophageal squamous cell precursor lesions and the clinicopathological correlations with the characteristics of esophageal squamous cell precursor lesions. Methods Florescence in situ hybridization was performed to detect hTERC amplification in different gradings of esophageal squamous cell precursor lesions. With retrospective follow-up data, clinicopathological correlations between hTERC and esophageal squamous cell precursor lesions were subjected to logistic regression analysis.

Results hTERC amplification gradually increased with upgrading of dysplasia, reaching the highest level in high-grade intraepithelial neoplasia, and there was a significant difference between the low-grade intraepithelial neoplasia group and the high-grade intraepithelial neoplasia group $(P=0.00)$. Logistic regression analysis showed that hTERC amplification was correlated with both dysplasia grading and ulcer characteristics of esophageal squamous cell precursor lesions $(P<0.05)$.

Conclusions hTERC amplification with increasing grading of esophageal squamous cell precursor lesions and the presence of ulcer characteristics might provide an important molecular and pathological marker for the diagnosis and clinical prognosis of esophageal squamous cell precursor lesions, especially for those ambiguous cases with more divergence in classification.
\end{abstract}

Keywords Esophageal $\cdot$ Premalignant lesions $\cdot$ hTERC $\cdot$ Dysplasia $\cdot$ Ulcer $\cdot$ Clinicopathological correlation

\section{Introduction}

The incidence of esophageal carcinoma has been increasing in recent years, making it the eighth most common malignancy and the sixth highest in terms of mortality worldwide [1]. There are approximately 400,000 newly diagnosed cases of esophageal carcinoma and approximately 300,000 related deaths worldwide each year, highlighting that this disease poses a serious threat to human health [2]. However, with ongoing advances in fiberoptic scopes to improve the detection rate of early-stage esophageal squamous cell carcinoma (ESCC), more cases of early-stage ESCC are being cured with surgery or radiotherapy; yet, most cases

Yanping $\mathrm{Hu}$

yphu92@163.com

1 Department of Pathology, Beijing LuHe Hospital, Capital Medical University, Beijing 101149, China

2 Department of Pathology, Beijing Friendship Hospital, Capital Medical University, Beijing 100050, China do not have obvious clinical symptoms, and most patients are diagnosed at an advanced stage of the disease, which is usually associated with a poor prognosis. Moreover, the prognosis of ESCC is still not as optimistic as other cancers of the digestive system, such as colorectal cancer. And the reported 5-year overall survival rate ranges from 15 to $40 \%$ $[3,4]$. Therefore, early diagnosis and treatment of ESCC is an effective and critical approach to improve the patient survival rate.

ESCC undergoes a similar progression as squamous cell carcinoma in other tissue types, with a multistage, multifactorial, and progressive carcinogenic process. As an important stage in the progression of ESCC, esophageal squamous cell precursor lesions (ESPLs) have become the most controversial topics of the current research in terms of precise pathological grading, molecular mechanism, clinical management, and prognosis, and ESPLs are observed frequently in clinical and pathological practice. The histopathological manifestations of ESPLs are structural abnormalities (i.e., structural destruction of squamous epithelium and loss of 
normal cellular polarity) and cytological abnormalities (i.e., irregular size and shape of squamous cells, deeply stained nucleus, increased ratio of nucleus to cytoplasm, and more mitotic figures). Based on a 13.5-year follow-up study with 682 cases, Wang GQ et al. [5] found that the overall incidence of ESPLs was approximately 114/682 (16.7\%), and ESPLs presented at various stages of squamous dysplasia, such as mild dysplasia (MID), moderate dysplasia (MOD), severe dysplasia (SD), and carcinoma in situ (CIS), with carcinogenesis rates of $2.9(1.6-5.2) \%, 9.8$ (5.3-18.3)\%, 28.3 $(15.3-52.3) \%$, and $34.4(16.6-71.4) \%$, respectively.

ESPLs are characterized by certain cytological and architectural changes in the esophageal epithelium. The World Health Organization (WHO) pathology and genetics classification system is the only one applied in China, and it is commonly used throughout the world. From the point of view of earlier studies, the squamous dysplasia mainly originates in the basal cells of squamous epithelium and undergoes histological and cytological microscopic changes from epithelial basal cells, progressing to the epidermal surface, until fulllayer squamous dysplasia has occurred. Most pathologists are aware that the characteristic pathological morphology should be classified as MOD or SD, and most subjectively perceive that these histological features have good repeatability. However, there is no general agreement among multidisciplinary fields regarding the recognition, histological classification, management, and prognosis of these challenging ESPLs. Therefore, it is necessary to identify a molecular marker for the accurate histopathological classification and subsequent proper clinical management of ESPLs.

Telomeres are short, tandem repetitive DNA sequences located at the ends of chromosomes that contain doublestranded TTAGGG DNA sequences with a single-stranded $3^{\prime}$ telomeric overhang. Telomerase is a ribonucleoprotein responsible for maintaining telomere length. The telomerase core has two components: catalytic telomerase reverse transcriptase (TERT) and telomerase RNA (TERC). TERT utilizes the template region ( $3^{\prime}$-CAAUCCCAAUC-5') of TERC to add TTAGGG DNA repeats and thereby extend single-stranded $3^{\prime}$ telomeric strands [6]. The human telomerase RNA (hTERC) gene is a cellular RNA-dependent DNA polymerase, a special type of reverse transcriptase. hTERC is the main component of telomerase, and it contains the template telomere DNA sequence and the telomerase protein-binding region. It also has the ability to reverse transcribe the human TTAGGG repeat sequence [7]. With the $3^{\prime}$ telomere as a primer, hTERC uses its own RNA as a template to synthesize telomeric DNA, whose function is to maintain telomere length at the end of chromosomes in eukaryotic cells, thereby protecting these chromosomal ends from being degraded or linked to other chromosomes [8]. In clinical studies, aggressive metastatic disease and poor prognosis have been correlated with high telomerase expression and activity in ovarian, breast, colorectal, prostate, and gastric cancer and melanoma [9], and some previous studies have shown the aberrant amplification of the hTERC gene in Barrett's esophagus [10] and ESCC [11, 12]. In this study, we investigated the hTERC expression profile in formalin-fixed, paraffin-embedded (FFPE) ESPL tissues and retrospectively analyzed the relationship between hTERC expression and clinicopathological characteristics of ESPL patients using detailed follow-up data.

\section{Materials and Methods}

\section{Study Population}

A total of 51 patients with ESPLs from Beijing Luhe Hospital, Capital Medical University, in Beijing, China, were enrolled in this study as the subject population for validation of hTERC expression (40 males (78.4\%) and 11 females (21.6\%); age range, 30-83 years; mean age, $60 \pm 13$ years). Diagnosis criteria were preferentially based on the WHO Classification System [5, 13], in which ESPLs are classified into two groups of intraepithelial neoplasia: lowgrade intraepithelial neoplasia (LGIEN) and high-grade intraepithelial neoplasia (HGIEN). If a diagnosis with two categories, such as LGIEN-HGIEN, was given, the higher grade was used in this study. In addition, 21 cases of ESCC and 21 cases of normal squamous esophageal epithelium (NS) were selected as the positive and negative control groups, respectively. According to the area of lymphatic infiltration, 51 cases were divided into three groups $(\leq 33 \%, 34-66 \%, \geq 67 \%)$. This study was approved by the Ethics Committee of Beijing Luhe Hospital of Capital Medical University, Beijing, China. Informed consent was waived by the Ethics Committee of Beijing Luhe Hospital of Capital Medical University as patients were identified retrospectively, according to institutional review board exempt protocols.

All patients included in the study met the following criteria: (1) initial ESPL patients with no previous history of ESCC; (2) esophageal mucosal biopsies were conducted using endoscopic procedures, and the sections were stained with hematoxylin and eosin (HE); (3) all cases were reviewed by three experienced pathologists in a joint consultation and grouped according to the consensus diagnosis; (4) complete clinicopathological information and follow-up data were available; and (5) a minimum follow-up of 1.5 years (or until progression to malignancy occurred) was conducted. In some cases, if the sample was taken from an improper site on suspicious esophageal mucosa, those cases diagnosed as invasive ESCC within 3 months after initial biopsy were excluded. Then, 51 cases were selected retrospectively from the pathology database from 2005 to 2018 in this study. The 
duration of the follow-up period was from 1.5 to 13 years, and the malignant transformation period was an average of 38 months (7-86 months).

In this study, hTERC gene amplification in ESPLs in four groups (NS, LGIEN, HGIEN, and ESCC) was statistically analyzed. Follow-up was performed at the third and sixth months after the initial diagnosis. Then, the patients with low-risk lesions and NS were followed up every 12 months, and those with high-risk lesions were followed up every 6 months.

\section{Amplification of hTERC by Fluorescence In Situ Hybridization (FISH)}

Esophageal mucosa tissue specimens were fixed with $10 \%$ buffered formalin for $12 \mathrm{~h}$, cleared in xylene, rehydrated in an alcohol gradient, conventionally embedded in paraffin, cut into $2-3-\mu \mathrm{m}$-thick slices, and affixed to anti-slip piece slides.

The hTERC detection procedure followed the instructions of the FISH detection kit. The slides were deparaffinized in xylene, digested with pepsin $\mathrm{K}$ for $8 \mathrm{~min}$, eluted with sodium chloride citrate dihydrate (SSC) at room temperature, dehydrated in an ethanol series (70\%, 85\%, and 100\%), and airdried. Then, the slides were placed in hybridization solution in a dark room, coverslipped, sealed with rubber sealant, denatured at $73{ }^{\circ} \mathrm{C}$ for $5 \mathrm{~min}$, and hybridized overnight at $42{ }^{\circ} \mathrm{C}$. The coverslip was removed, and the slides were incubated three times in $50 \% 2 \times$ SSC solution for $10 \mathrm{~min}$ each at $46{ }^{\circ} \mathrm{C}$, once in $2 \times \mathrm{SSC}$ solution for $10 \mathrm{~min}$, and once in $2 \times \mathrm{SSC} / 0.1 \%$ Nonidet P-40 solution for $5 \mathrm{~min}$; then, the slides were rinsed for $3 \mathrm{~min}$ in $70 \%$ ethanol, air-dried at room temperature, stained with 4,6-diamidino-2-phenylindole (DAPI), coverslipped, and observed under a fluorescence microscope (Zeiss Imager M2 AX10).

\section{hTERC FISH Signal Scoring and Evaluation}

The hTERC FISH detection kit (Cervical cells kit, Beijing GP Medical Technologies, Ltd., Beijing, China) includes the GLP TERC/CSP3 probe group. The chromosome probe 3q26.3 (hTERC) is labeled with the SpectrumRed fluorophore and has the sequence 5'-CUAACCCUAAC-3'; the CSP3 probe is labeled with the SpectrumGreen fluorophore and has a proprietary sequence. The control CSP3 probe hybridizes with the 3p11.1-q11.1 region of the centromere in chromosome 3 . There were 2 red and 2 green hybridization signals in each interphase nucleus of cells without hTERC gene amplification. At least 2 green signals and $>2$ red signals should be detected in each interphase nucleus of cells with hTERC gene amplification.

FISH results were evaluated according to the kit instructions: (1) normal: 2 red and green signals in a single interphase nucleus; (2) abnormal/hTERC gene amplification: $>2$ red signals and $\geq 2$ green signals in a single interphase nucleus. One hundred cells were randomly observed in each case, and the percentage of abnormal hTERC signals was determined. The abnormal threshold was calculated using the equation average $+3 \times$ standard deviation to be $7 \%$ according to the positive threshold mentioned in the kit (cervical cell TERC site amplification kit) in this experiment. A total of 100 cell nuclei were randomly counted in each sample. If the percentage of cells with an abnormal signal was higher than the abnormal threshold, then the sample was characterized as positive; if the percentage was lower than the abnormal threshold, then the sample was characterized as negative; if the percentage was equal to the abnormal threshold, then more cells were analyzed to obtain the final result.

All slides were observed independently by two experienced pathologists, and the results were reached by consensus with a third more senior pathologist if necessary. All reviewers were blinded to ESPL clinical information.

\section{Statistical Analysis}

Statistical analysis was performed using SPSS version 19.0 (SPSS for Windows, SPSS Inc., Chicago, IL, USA). Then, one-way ANOVA and the Chi-square test were used to analyze significant differences in hTERC amplification between the different ESPL groups and the control groups (NS and ESCC groups). Univariate analysis and logistic regression were performed to analyze the correlations between hTERC expression and clinicopathological characteristics of ESPLs. $P<0.05$ indicated a statistically significant difference.

\section{Results}

\section{Patient Demographics}

A total of 51 cases were divided into two groups based on two classification systems: 25 cases of LGIEN and 26 cases of HGIEN. The mean age of the 51 ESPL patients was $60 \pm 12$ years (range $30-83$ years), and 40 males $(78.4 \%$ ) and 11 females (21.6\%) were included. There were 25 cases of ESPLs with ulcers (49.0\%). According to the extent of lymphocytic infiltration in mucosal tissues, all 51 cases of ESPLs were divided into two groups: 40 cases of less than one-third infiltration (78.4\%) and 11 cases of less than twothird infiltration (21.6\%). The proportion of patients with a smoking history was 51.0\% (26/51), and 37.3\% (19/51) had a drinking habit. The overall carcinoma transformation rate of ESPLs was 25.5\% (13/51). The distribution of clinicopathological characteristics of all 93 cases, including 21 of 
NS and 21 of ESCC, is provided in Table 1. The pathological morphology of the ESPLs is shown in Fig. 1a, c, e.

\section{Amplification of hTERC}

All 93 FFPE samples were subjected to hTERC gene amplification analysis. After FISH analysis, the 93 cases were characterized as positive or negative using the threshold value. The overall amplification of hTERC in ESPLs was $43.1 \%$ (22/51). Compared with the 21 cases of NS and 21 cases of ESCC, hTERC amplification in ESPL samples gradually increased with advancing dysplasia grade, reaching the highest level in HGIEN. Moreover, hTERC amplification was not found in any NS cases but was detected in all ESCC cases $(100 \%)$. hTERC gene amplification in ESPLs in four

Table 1 Demographics and clinicopathological parameters of all 93 cases

\begin{tabular}{|c|c|c|c|c|}
\hline \multirow[t]{2}{*}{ Group } & \multicolumn{4}{|c|}{ Characteristics } \\
\hline & NS (\%) & LGIEN (\%) & HGIEN (\%) & $\operatorname{ESCC}(\%)$ \\
\hline Total $(\%)$ & $21(100)$ & $25(100)$ & $26(100)$ & $21(100)$ \\
\hline \multicolumn{5}{|l|}{ Gender } \\
\hline Female & $12(57.1)$ & $6(24.0)$ & $5(19.2)$ & $3(14.3)$ \\
\hline Male & $9(42.9)$ & $19(76.0)$ & $21(80.8)$ & $18(85.7)$ \\
\hline Mean age & $56 \pm 12$ & $56 \pm 14$ & $63 \pm 10$ & $63 \pm 9$ \\
\hline \multicolumn{5}{|l|}{ Site } \\
\hline$\leq 24 \mathrm{~cm}$ & $0(0)$ & $4(16.0)$ & $5(19.2)$ & $1(4.8)$ \\
\hline $25-32 \mathrm{~cm}$ & $0(0)$ & $6(24.0)$ & 7 (26.9) & $12(57.1)$ \\
\hline $33-40 \mathrm{~cm}$ & $21(100)$ & $12(48.0)$ & $9(34.7)$ & $7(33.3)$ \\
\hline Other & $0(0)$ & $3(12.0)$ & $5(19.2)$ & $1(4.8)$ \\
\hline \multicolumn{5}{|l|}{ Ulcer } \\
\hline No & $21(100)$ & $18(72.0)$ & $8(30.8)$ & $5(23.8)$ \\
\hline Yes & $0(0)$ & $7(28.0)$ & $18(69.2)$ & $16(76.2)$ \\
\hline \multicolumn{5}{|c|}{ Lymphocyte infiltration } \\
\hline$\leq 33 \%$ & $20(95.2)$ & $21(84.0)$ & $19(73.1)$ & $6(28.6)$ \\
\hline $34-66 \%$ & $1(4.8)$ & $4(16.0)$ & 7 (26.9) & $11(52.4)$ \\
\hline$\geq 67 \%$ & $0(0)$ & $0(0)$ & $0(0)$ & $4(19.0)$ \\
\hline \multicolumn{5}{|c|}{ Pack-years smoked } \\
\hline None & $14(66.7)$ & $12(48.0)$ & $13(50.0)$ & $9(42.9)$ \\
\hline$\leq 40$ & $5(23.8)$ & $6(24.0)$ & 7 (26.9) & $2(9.5)$ \\
\hline$>40$ & $2(9.5)$ & $7(28.0)$ & $6(23.1)$ & $10(47.6)$ \\
\hline \multicolumn{5}{|l|}{ Drinking habit } \\
\hline No & $19(90.5)$ & $17(68.0)$ & $15(57.7)$ & $9(42.9)$ \\
\hline Yes & $2(9.5)$ & $8(32.0)$ & $11(42.3)$ & $12(57.1)$ \\
\hline \multicolumn{5}{|l|}{ Prognosis } \\
\hline None & $21(100)$ & $21(84.0)$ & $17(65.4)$ & \\
\hline Progression & $0(0)$ & $4(16.0)$ & 9 (34.6) & \\
\hline
\end{tabular}

NS normal squamous esophageal epithelium; LGIEN low-grade intraepithelial neoplasia; HGIEN high-grade intraepithelial neoplasia; ESCC esophageal squamous cell carcinoma

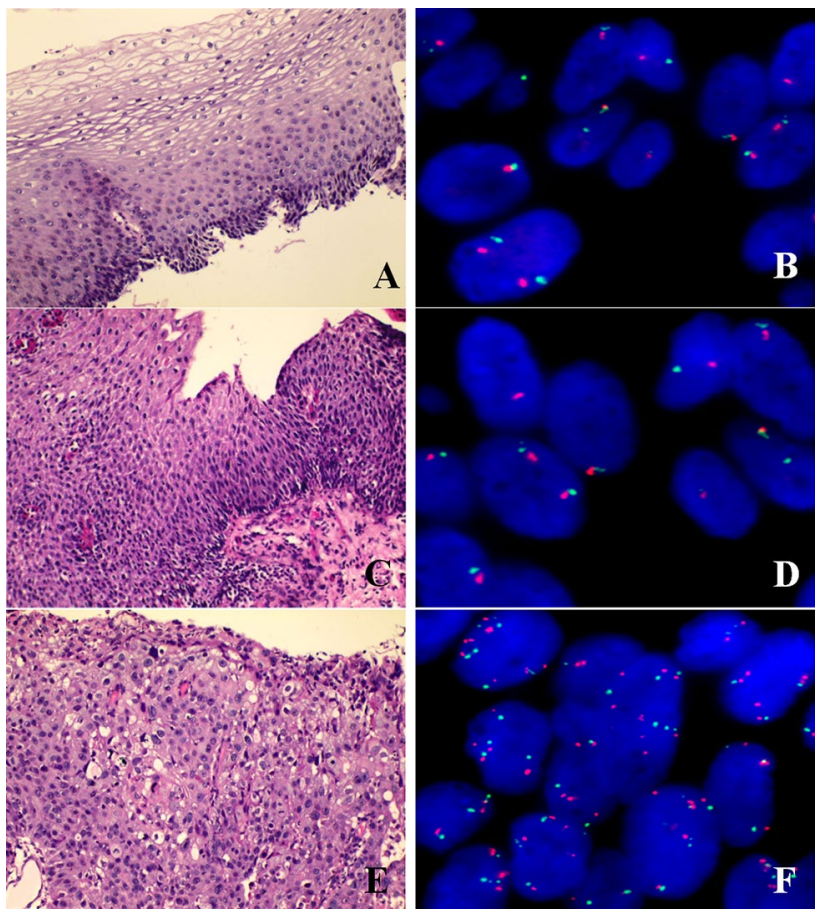

Fig. 1 a Normal squamous esophageal epithelium (NS); HE $\times 100$. b NS; CEP3: hTERC, 2: 2; FISH $\times 1000$. c Low-grade intraepithelial neoplasia (LGIEN); HE $\times 100$. d LGIEN; CEP3: hTERC, 2: 2; FISH $\times 1000$. e High-grade intraepithelial neoplasia (HGIEN); HE $\times 100$. f HGIEN; CEP3: hTERC, 2: 3; FISH $\times 1000$

groups (NS, LGIEN, HGIEN, and ESCC) was statistically analyzed.

Amplification of the hTERC gene gradually increased with the degree of dysplasia in ESPLs, and there were significant differences among the six groups $(P=0.00)$ and among the four groups $(P=0.00)$. Furthermore, within the four groups, there was a significant difference between most pairs of adjacent groups: LGIEN and HGIEN $(P=0.00)$ and HGIEN and ESCC $(P=0.01)$ but not NS and LGIEN $(P=0.29)$. The summary of differential hTERC amplification in 51 cases of ESPLs and the control groups as determined by FISH is shown in Table 2. The histological observations of hTERC amplification in ESPL cases and controls by FISH microscopy are shown in Fig. 1b, d, f.

\section{Correlation of hTERC Amplification with Clinicopathological Parameters in ESPLs}

To further analyze the correlation between hTERC amplification and clinicopathological characteristics of ESPL cases, logistic regression was subsequently performed. As shown in Table 2 and Fig. 1, hTERC gene amplification increased with more advanced dysplasia grade, and the trends of increased hTERC amplification also correlated with dysplasia grade in the logistic regression analysis $(P=0.00)$. 
Table 2 Amplification of hTERC in ESPLs by FISH

\begin{tabular}{lllll}
\hline Group & $n(\%)$ & hTERC & & \\
\cline { 3 - 5 } & & Positive, $n(\%)$ & Negative, $n(\%)$ & $P$ \\
\hline NS & $21(22.6)$ & $0(0)$ & $21(100)$ & \\
LGIEN & $25(26.9)$ & $3(12.0)$ & $22(88.0)$ & \\
HGIEN & $26(27.9)$ & $19(73.1)$ & $7(26.9)$ & $0.00^{*}$ \\
ESCC & $21(22.6)$ & $21(100)$ & $0(0)$ & $0.01^{*}$ \\
Total & $93(100)$ & $43(46.2)$ & $50(53.8)$ & $0.00^{*}$ \\
\hline
\end{tabular}

$N S$ normal squamous esophageal epithelium; LGIEN low-grade intraepithelial neoplasia; HGIEN high-grade intraepithelial neoplasia; ESCC esophageal squamous cell carcinoma

$* P<0.05$ : All groups of ESPLs $(P=0.00)$; LGIEN versus HGIEN $(P=0.00)$; HGIEN versus ESCC $(P=0.01)$

Furthermore, the ratio of cells with hTERC amplification was significantly higher in ESPL cases with ulcers than in cases without ulcers $(P=0.02)$. Otherwise, hTERC amplification showed no significant difference based on any other clinicopathological parameter, e.g., age, ESPL site in the esophagus, smoking or drinking habit, lymphocyte infiltration in mucosal intrinsic stroma of ESPLs, and carcinoma transformation $(P>0.05)$. Logistic regression analysis of hTERC amplification with clinicopathological parameters of ESPLs is shown in Table 3.

\section{Discussion}

In the 2000 WHO classification [14], a two-tier system was initially introduced to replace the classical three-tier system of MID, MOD, and SD/CIS. The views of Japanese and Western pathologists have differed significantly regarding the pathological criteria of squamous dysplasia and ESCC. Before the term of intraepithelial neoplasia of the esophagus was introduced in the $2000 \mathrm{WHO}$ classification [14], SD diagnosed according to the WHO classification was usually equivalent to CIS or noninvasive ESCC diagnosed by Japanese pathologists. Moreover, one particular situation is the presence of basal layer-type CIS, which is often underdiagnosed as low-/high-grade intraepithelial neoplasia by Western pathologists. In the 2010 WHO classification [12], the lower portion of MOD was grouped with MID to form the LGIEN group. The upper portion of MOD was grouped with SD/CIS to form the HGIEN group. To a large extent, these changes simplified the classification system for ESPLs and reduced the divergence among pathologists. While dealing with MOD, pathologists need to classify the lower and the upper portions into LGIEN and HGIEN according to the morphology. Then, there is inevitably subjective variability in the pathological classification. Additionally, because HGIEN ranges from some upper portion of MOD to CIS,
Table 3 Correlations of hTERC amplification with clinicopathological parameters and the prognosis of 51 ESPL cases

\begin{tabular}{|c|c|c|c|c|}
\hline Parameters & hTERC & Reg. coeff. & $P$ value & OR $(95 \% \mathrm{CI})$ \\
\hline \multicolumn{5}{|c|}{ Pack-years smoked } \\
\hline None & $10(45.5)$ & Reference & & 1 \\
\hline$\leq 40$ & $7(31.8)$ & 0.56 & 0.42 & $1.75(0.45-6.77)$ \\
\hline$>40$ & $5(22.7)$ & -0.06 & 0.93 & $0.94(0.24-3.71)$ \\
\hline \multicolumn{5}{|l|}{ Drinking habit } \\
\hline No & $12(54.5)$ & Reference & & \\
\hline Yes & $10(45.5)$ & 0.62 & 0.29 & $1.85(0.59-5.85)$ \\
\hline \multicolumn{5}{|c|}{ Grade of dysplasia } \\
\hline LGIEN & $3(13.6)$ & Reference & & 1 \\
\hline HGIEN & $19(86.4)$ & 19.38 & $0.00 *$ & $\begin{array}{l}19.905(4.507- \\
87.904)\end{array}$ \\
\hline \multicolumn{5}{|l|}{ Ulcer } \\
\hline No & $7(31.8)$ & Reference & & 1 \\
\hline Yes & $15(68.2)$ & 1.40 & $0.02 *$ & $4.07(1.25-13.24)$ \\
\hline \multicolumn{5}{|c|}{ Lymphocyte infiltration } \\
\hline$\leq 33 \%$ & $3(13.6)$ & Reference & & 1 \\
\hline $34-66 \%$ & $13(59.1)$ & 1.63 & $0.03 *$ & $5.12(1.15-22.73)$ \\
\hline$\geq 67 \%$ & $6(27.3)$ & 1.65 & 0.06 & $5.20(0.92-29.26)$ \\
\hline \multicolumn{5}{|c|}{ Carcinoma transformation } \\
\hline None & $14(63.6)$ & Reference & & 1 \\
\hline Progression & $8(36.4)$ & 1.01 & 0.13 & $2.74(0.75-10.04)$ \\
\hline
\end{tabular}

$* P<0.05$

both clinicians and pathologists still consider that the upper part of MOD should be treated differently from CIS, the latter of which is considered to require more aggressive treatment strategies. Therefore, in most Chinese hospitals, although the two-level classification criteria were adopted, most pathologists are still accustomed to adding an explicit suffix category, such as "MOD-SD" or "CIS," as supplementary instructions to facilitate better guidance with proper management and prognosis, especially when dealing with grading of precursor oral, laryngeal, and esophageal lesions. Therefore, there are still some issues that need to be resolved in terms of the exact pathological classification of esophageal squamous epithelial lesions [15].

The most prominent clinical feature of ESPLs is the instability of bidirectional development: ESPLs can either progress into ESCC or remain unchanged at one stage for many years until they return to normal. Skacel et al. [16] analyzed the prognosis of esophageal low-grade intraepithelial lesions using 2-year and 6-month follow-up data of 25 cases and found that $60 \%$ naturally subsided, $12 \%$ remained at the original grade, and $28 \%$ progressed to high-grade intraepithelial lesions or ESCC. As the degree of dysplasia in the esophageal squamous epithelium increases, the risk of progression to ESCC also increases. Therefore, appropriate clinical treatment and management should be adopted 
according to different classifications of ESPLs. However, in clinical and pathological practice, the precise grading and histopathological diagnosis of ESPLs, especially for MOD or SD lesions, inevitably remains controversial. Wang GQ et al. [5] and the 2010 WHO Classification of Tumours of the Digestive System [10] both showed that SD and CIS have a similar risk of progression to ESCC and should be grouped together for clinical management. This finding may be due to the presence of residual squamous epithelium on the mucosal surface of the esophagus or incomplete histological structure of the squamous epithelium in the endoscopic biopsy tissue, which may confuse the pathological grading of ESPLs regarding whether squamous cell dysplasia exceeds one-half of the epithelium or covers the full thickness.

Telomeres have a capping function in which they protect the ends of chromosomes from degradation and fusion [17]. Telomerase activity is temporally regulated in accordance with organismal development; it is detected at the fetal and newborn stages but gradually declines to extremely low or absent levels thereafter, except in cells with higher regenerative proliferation, including hematopoietic, epidermal, and gastrointestinal cells [9, 18, 19]. Hsu et al. [11] found that the hTERT and hTERC genes were expressed in $64.9 \%$ and $79.7 \%$ of 74 specimens of ESCC, respectively. Even though telomerase expression is not related to tumor stages and prognosis, the cumulative 4-year survival rates of telomerase-positive and telomerase-negative cases were $35.9 \%$ and $31.2 \%$, respectively $(P=0.84)$. In this study, hTERC gene amplification gradually increased with the severity of dysplasia in ESPLs and showed significant differences between the LGIEN (12.0\%) and HGIEN (73.1\%) groups in the two-tier classification, showing an approximately sixfold increase in hTERC amplification. These findings indicated that hTERC might be a valuable molecular marker for assisting with the pathological classification of ESPLs, especially for discriminating LGIEN and HGIEN. Moreover, during routine pathological practice, the pathologist could refer to hTERC expression to guide grading when encountering histological morphology with more divergence in indeterminate ESPLs between LGIEN and HGIEN. The existence of hTERC amplification may be indicative of high-risk lesions, and some pathologists would classify specimens with hTERC amplification as HGIEN; in contrast, no hTERC amplification may suggest low-risk lesions, and some specimens without hTERC amplification are more likely to be classified as LGIEN, thus enhancing the confidence of pathologists in the auxiliary classification of controversial cases of ESPLs and facilitating more appropriate and effective clinical treatment.

The altered expression of telomerase could illustrate its potential applications in diagnostic and prognostic assessments and clinical treatment, and it is expected to become a valuable molecular marker in clinicopathology. The distinct phenotypic disparity between different groups of ESPLs creates the potential for hTERC to serve as a universal cancer biomarker for disease diagnosis and prognosis. Previous studies have shown a potential correlation between high telomerase expression and poor prognosis and aggressive metastasis of some malignancies, including ovarian, breast, prostate, liver and colorectal cancer, and melanoma [9, 20-23]. Moreover, there is a valuable correlation between telomerase expression and clinical factors related to drug resistance in breast [24] and gastric [25] cancer. Parallel in vitro studies have confirmed that TERT overexpression is associated with resistance to radiation and imatinib [26, 27]. However, studies concerning the clinicopathological relationship between hTERC dysregulation and clinicopathological features of ESPLs are rare. Combined with the exhaustive follow-up data on ESPLs, a comprehensive clinicopathological analysis explored the possible associations between hTERC expression and clinicopathological characteristics of ESPLs.

In our univariate analysis, two clinicopathological factors, pathological classification and ulcers, showed significant differences regarding hTERC amplification. As previously mentioned, hTERC amplification is correlated with both increased heteromorphism and the presence of ulcers in ESPLs. Subsequent logistic analysis further confirmed a statistically significant difference in hTERC amplification between LGIEN (12.0\%) and HGIEN (73.1\%); moreover, there was a higher rate of hTERC amplification in ESPLs with ulcers $(68.2 \%)$ than in those without ulcers (31.8\%). It is speculated that there might be some possible correlations between the factors of ulceration and hTERC amplification, which might stimulate the inflammatory factors and promote the progression of ESPLs into ESCC. Whether all of these speculations are reasonable or not, large sample studies are still necessary for further verification. In addition to these two parameters, hTERC amplification showed no statistical correlation with any other clinicopathological parameter of ESPLs. Although a difference in hTERC amplification between LGIEN and HGIEN was found in this study, we still did not find a clinicopathological correlation between hTERC and the progression of ESPLs. The reasons may be manifold: The sample size of the current study may be not sufficient to achieve a convincing conclusion, and the research designed here still needs further refinement, optimization, and improvement. However, a potential application value of the hTERC gene for classifying ESPLs was inferred.

Briefly, the current preliminary study potentially indicates that hTERC expression may be one of the factors related to the classification of pathological ulcers, which may be involved in the carcinogenic inflammatory mechanisms in ESPLs. In terms of the relationship between 
hTERC expression and ulcerative factors associated with ESPLs, the underlying mechanism of hTERC in this process remains unclear. There may be synergistic effects or interactions between inflammatory factors and the hTERC gene in the pathogenesis or progression of ESPLs, but these speculations require further in-depth experimental research to clarify. The relationship between hTERC and pathological ulcers may provide important clues and practical significance to perfect the pathological classification system used to guide clinical management and prognosis of ESPLs and to fundamentally reduce the incidence and progression of early ESCC. It is necessary to fully understand the role of hTERC in the molecular mechanism and characteristics of ESPL development for digestive system pathologists to reasonably assess the prognosis of ESPLs and to implement appropriate and effective treatment.

Funding This study was supported by a grant from the Science and Technology Project Fund of Tongzhou District in Beijing (KJ2016CX037).

\section{Compliance with ethical standards}

Conflict of interest The authors declare that they have no conflicts of interest.

Open Access This article is distributed under the terms of the Creative Commons Attribution-NonCommercial 4.0 International License (http://creativecommons.org/licenses/by-nc/4.0/), which permits any noncommercial use, distribution, and reproduction in any medium, provided you give appropriate credit to the original author(s) and the source, provide a link to the Creative Commons license, and indicate if changes were made.

\section{References}

1. Kamangar F, Dores GM, Anderson WF. Patterns of cancer incidence, mortality, and prevalence across five continents: Defining priorities to reduce cancer disparities in different geographic regions of the world. J Clin Oncol. 2006;24:2137-2150.

2. Guo JH, Xing GL, Fang XH, et al. Proteomic profiling of fetal esophageal epithelium, esophageal cancer, and tumor-adjacent esophageal epithelium and immunohistochemical characterization of a representative differential protein, PRX6. World J Gastroenterol. 2017;23:1434-1442.

3. Ohashi S, Miyamoto S, Kikuchi O, Goto T, Amanuma Y, Muto M. Recent advances from basic and clinical studies of esophageal squamous cell carcinoma. Gastroenterology. 2015;149:1700-1715.

4. Jesinghaus M, Steiger K, Slotta-Huspenina J, et al. Increased intraepithelial CD3 + T-lymphocytes and high PD-L1 expression on tumor cells are associated with favorable prognosis in esophageal squamous cell carcinoma and allow prognostic immunogenic subgrouping. Oncotarget. 2017;8:46756-46768.

5. Wang GQ, Abnet CC, Shen Q, et al. Histological precursors of oesophageal squamous cell carcinoma: results from a 13 year prospective follow up study in a high risk population. Gut. 2005;54:187-192.
6. Morin GB. The human telomere terminal transferase enzyme is a ribonucleoprotein that synthesizes TTAGGG repeats. Cell. 1989;59:521-529.

7. Fu D, Collins K. Purification of human telomerase complexes identifies factors involved in telomerase biogenesis and telomere length regulation. Mol Cell. 2007;28:773-785.

8. Lewis KA, Tollefsbol TO. Regulation of the telomerase reverse transcriptase subunit through epigenetic mechanisms. Front Genet. 2016;9:83.

9. Xu Y, Goldkorn A. Telomere and telomerase therapeutics in cancer. Genes (Base). 2016;7:E22.

10. Wang J, Wang LP, Xu S, Yang GZ. Morphology, immunohistochemistry and hTERC gene in situ hybridization in Barrett's esophagus. Zhonghua Bing Li Xue Za Zhi. 2013;42:4-9.

11. Hsu CP, Lee LW, Shai SE, Chen CY. Clinical significance of telomerase and its associate genes expression in the maintenance of telomere length in squamous cell carcinoma of the esophagus. World J Gastroenterol. 2005;11:6941-6947.

12. Wang YF, Wang XS, Gao SG, et al. Clinical significance of combined detection of human papilloma virus infection and human telomerase RNA component gene amplification in patients with squamous cell carcinoma of the esophagus in northern China. Eur J Med Res. 2013;18:11.

13. Bosman FT, Carneiro F, Hruban RH, Theise ND. WHO Classification of Tumours of the Digestive System. Lyon: IARC Press; 2010:22-23.

14. Hamilton Stanley R, Aaltonen Lauri A. World Health Organization Classification of Tumours. Pathology and Genetics. Tumours of the Digestive System. Lyon: IARC Press; 2000:15-16.

15. Shimizu M, Nagata K, Yamaguchi H, Kita H. Squamous intraepithelial neoplasia of the esophagus: Past, present, and future. $J$ Gastroenterol. 2009;44:103-112.

16. Skacel M, Petras RE, Gramlich TL, Sigel JE, Richter JE, Goldblum JR. The diagnosis of low-grade dysplasia in Barrett's esophagus and its implications for disease progression. Am J Gastroenterol. 2000;95:3383-3387.

17. Griffith JD, Comeau L, Rosenfield S, et al. Mammalian telomeres end in a large duplex loop. Cell. 1999;97:503-514.

18. Wright WE, Piatyszek MA, Rainey WE, Byrd W, Shay JW. Telomerase activity in human germline and embryonic tissues and cells. Dev Genet. 1996;18:173-179.

19. Forsyth NR, Wright WE, Shay JW. Telomerase and differentiation in multicellular organisms: turn it off, turn it on, and turn it off again. Differentiation. 2002;69:188-197.

20. Oishi T, Kigawa J, Minagawa Y, Shimada M, Takahashi M, Terakawa N. Alteration of telomerase activity associated with development and extension of epithelial ovarian cancer. Obstet Gynecol. 1998;91:568-571.

21. Swellam M, Ismail M, Eissa S, Hamdy M, Mokhtar N. Emerging role of p53, bcl-2 and telomerase activity in Egyptian breast cancer patients. IUBMB Life. 2004;56:483-490.

22. Xie XC, Ge LY, Lai H, Qiu H, Tang F, Qin YZ. The relationship between telomerase activity and clinicopathological parameters in colorectal cancer: A meta-analysis. Balkan Med J. 2016;33:64-671.

23. Pirker C, Holzmann K, Spiegl-Kreinecker S, et al. Chromosomal imbalances in primary and metastatic melanomas: overrepresentation of essential telomerase genes. Melanoma Res. 2003;13:483-492.

24. Gay-Bellile M, Romero P, Cayre A, et al. ERCC1 and telomere status in breast tumours treated with neoadjuvant chemotherapy and their association with patient prognosis. J Pathol Clin Res. 2016;2:234-246.

25. Wang L, Li PF, Geng M, Cao YC, Yin YC. Correlation between chemosensitivity to anticancer drugs and telomerase reverse 
transcriptase mRNA expression in gastric cancer. Diagn Pathol. 2013;22:33.

26. Deville L, Hillion J, Pendino F, Samy M, Nguyen E, SégalBendirdjian E. hTERT promotes imatinib resistance in chronic myeloid leukemia cells: therapeutic implications. Mol Cancer Ther. 2011;10:711-719.
27. Xu B, Li C, Sung C. Telomerase inhibitory effects of medicinal mushrooms and lichens, and their anticancer activity. Int $\mathrm{J}$ Med Mushrooms. 2014;16:17-28. 